\title{
ON THE TRANSCENDENCE DEGREE OF GROUP ALGEBRAS OF NILPOTENT GROUPS
}

\author{
by MARTIN LORENZ*
}

(Received 7 January, 1983)

Introduction. Let $G$ be a finitely generated (f.g.) torsion-free nilpotent group. Then the group algebra $k[G]$ of $G$ over a field $k$ is a Noetherian domain and hence has a classical division ring of fractions, denoted by $k(G)$. Recently, the division algebras $k(G)$ and, somewhat more generally, division algebras generated by f.g. nilpotent groups have been studied in [3] and [5]. These papers are concerned with the question to what extent the division algebra determines the group under consideration. Here we continue the study of the division algebras $k(G)$ and investigate their Gelfand-Kirillov (GK-) transcendence degree.

Recall that the $G K$-transcendence degree of an associative $k$-algebra $A$ is defined by

$$
\operatorname{Tdeg}_{k}(A)=\sup _{V} \inf _{b} d_{k}(k[b V]),
$$

where the supremum is taken over the finite-dimensional $k$-subspaces $V$ of $A$, the infimum is taken over the regular elements $b \in A$, and $d_{k}($.$) denotes GK-dimension over$ $k$. For a detailed discussion of the invariants $\operatorname{Tdeg}_{k}($.$) and d_{k}($.$) , in particular the latter,$ we refer to [2].

In Section 1, we show that, for $G$ f.g. torsion-free nilpotent, one has $\operatorname{Tdeg}_{k}(k(G))=$ $\operatorname{Tdeg}_{k}(k[G])$. Here, the inequality $\operatorname{Tdeg}_{k}(k(G)) \leq \operatorname{Tdeg}_{k}(k[G])$ is clear from the definition of Tdeg (take $b$ to be a common denominator for $V$ ); so the interest lies in the reverse inequality. We further define a purely group theoretical invariant, $t(G)$, and show that $\operatorname{Tdeg}_{k}(k(G))=t(G)$, thereby reducing the problem of explicitly calculating $\operatorname{Tdeg}_{k}(k(G))$ to group theory. Our main technical tool is the so-called Hilbert-Neumann construction which has been extensively used in [3] and [5]. The results in this section are of a fairly general nature. All that is needed here is the fact that $G$ is an ordered group and that (twisted) group algebras of $G$ have classical rings of fractions.

In Section 2, we show that, for $G$ nilpotent of class at most two, the invariant $t(G)$ equals the usual growth degree $d(G)$ of $G$ (see [1]). In particular, since $d(G)=d_{k}(k[G])$, we obtain that $\operatorname{Tdeg}_{k}(k(G))=\operatorname{Tdeg}_{k}(k[G])=d_{k}(k[G])$ for $G$ f.g. torsion-free nilpotent of class at most two. Presumably, the equality $t(G)=d(G)$ holds for all f.g. nilpotent groups, but we have been unable to confirm this. We also include some related results on $t($.$) and$ on the GK-dimension of skew polynomial rings.

1. Filtrations and graduations. Throughout this section, $D$ will denote a division algebra over a field $k, G$ will be any ordered group, and $S=D *((G)$ ) will be a (crossed) Hilbert-Neumann algebra of $G$ over $D$. Thus each element $s \in S$ can be uniquely written

\footnotetext{
* Research supported by the Deutsche Forschungsgemeinschaft, Grant No. Lo 261/2-1.
}

Glasgow Math. J. 25 (1984) 167-174. 
as a formal sum

$$
s=\sum_{x \in G} s_{x} \bar{x}
$$

with $s_{x} \in D$ and with Supp $s=\left\{x \in G \mid s_{x} \neq 0\right\}$ a well-ordered subset of $G$. Addition of such elements is defined componentwise and the multiplication of $S$ is given by

$$
\sum_{x \in G} s_{x} \bar{x} \cdot \sum_{y \in G} t_{y} \bar{y}=\sum_{z \in G}\left(\sum_{x y=z} s_{x} t_{y}^{\alpha(x)-1} \gamma(x, y)\right) \bar{z} \text {. }
$$

Here, $\gamma: G \times G \rightarrow D^{*}=D \backslash\{0\}$ and $\alpha: G \rightarrow \operatorname{Aut}_{k-a l g}(D)$ are maps which, of course, have to satisfy certain relations in order to make this multiplication associative (see $[4,1.1]$ ). Without loss of generality, we will assume that $\overline{1}$ is the identity of $S$ and we will identify each $d \in D$ with $d \overline{1} \in S$, thus viewing $D$ as a subalgebra of $S$. By the Malcev-Neumann theorem, $S$ is a division algebra (see [7, Theorem 13.2.11]). We let

$$
\nu: S \rightarrow G \cup\{\infty\} \text { with } \nu(0)=\infty \text { and } \nu(s)=\min \operatorname{Supp} s \quad(s \neq 0)
$$

denote the lowest term valuation of $S$.

Now let $R$ be a $k$-subalgebra of $S$ with $D \subseteq R$. For each $x \in G$, set

$$
R_{x}=\{r \in R \mid \nu(r) \geq x\} \text {, and } R_{x+}=\{r \in R \mid \nu(r)>x\}=\bigcup_{y>x} R_{y} .
$$

These are $D$-subspaces of $R$ with $R_{y} \subseteq R_{x}$ for $x \leq y$ and $R_{x} R_{y} \subseteq R_{x y}$, and similarly for $R_{x+}$ and $R_{y+\cdot}$. Also, $\bigcup_{x \in G} R_{x}=R$ and $\bigcap_{x \in G} R_{x}=\{0\}$. The system $\left\{R_{x} \mid x \in G\right\}$ will be called the $G$-filtration of $R$. As usual, we define the associated $G$-graded algebra of $R$ by

$$
\text { gr } R=\bigoplus_{x \in G} R_{x} / R_{x+\cdot}
$$

Multiplication in gr $R$ is given by

$$
\left(r+R_{x+}\right)\left(t+R_{y+}\right)=r t+R_{x y+} \in R_{x y} / R_{x y+}
$$

for $r \in R_{x}, t \in R_{y}$. Now set

$$
G_{R}=\nu(R \backslash\{0\}) \subseteq G .
$$

Then $G_{R}$ is a subsemigroup of $G$ and, clearly, $R_{x} / R_{x+}$ is nonzero if and only if $x \in G_{R}$. More precisely, we have the following result.

LEMMA 1.1. With the above notation, we have $\operatorname{gr} R=D * G_{R}$, a crossed product of $G_{R}$ over $D$ obtained by restricting the defining data $\left(\gamma\right.$ and $\alpha$ ) of $S=D^{*}((G))$ from $G$ to $G_{R}$.

Proof. Let $x \in G_{R}$. Then there exists $r=r_{x} \bar{x}+r_{+} \in R$, where $r_{x} \in D, r_{x} \neq 0$ and $\nu\left(r_{+}\right)=-x$. Since $D \subseteq R$ we can assume that $r_{x}=1$. Therefore, if $s+R_{x+} \in R_{x} / R_{x+}$ is arbitrary, say $s=s_{x} \bar{x}+s_{+}$with $\nu\left(s_{+}\right)>x$ then $s \equiv s_{x} r\left(\bmod R_{x+}\right)$. This shows that $R_{x} / R_{x+}$ is onedimensional over $D$, generated by $\dot{x}=\bar{x}+R_{x+}$, and each element of gr $R$ can be uniquely written as a finite sum $\sum_{x \in G_{R}} d_{x} \dot{x}$, with $d_{x} \in D$. One easily verifies the relations $\dot{x} \cdot \dot{y}=$ 
$\gamma(x, y) \dot{x y}$ and $d \dot{x}=\dot{x} d^{\alpha(x)}$ for $x, y \in G_{R}$ and $d \in D \cong R_{1} / R_{1+}$, where $\gamma$ and $\alpha$ are the defining data of $S$. This proves the lemma.

Our next lemma compares the GK-transcendence degrees of $R$ and of gr $R$ over $k$. For $r \in R$ with $\nu(r)=x \in G$, we set $\tilde{r}=r+R_{x+} \in R_{x} / R_{x+}$. Note that in gr $R$ we have $\tilde{r}_{1} \cdot \tilde{r}_{2}=\widetilde{r_{1} r_{2}}$.

LEMMA 1.2. With the above notation, $\operatorname{Tdeg}_{k}(R) \geq \operatorname{Tdeg}_{k}(\operatorname{gr} R)$.

Proof. Let $W \subseteq$ gr $R$ be a finite-dimensional $k$-subspace and choose a finitedimensional $k$-subspace $V \subseteq R$ such that gr $V$ contains $W$, where

$$
\text { gr } V=\langle\tilde{r} \mid r \in V \backslash\{0\}\rangle_{k \text {-space }} \subseteq \text { gr } R \text {. }
$$

Let $s \in R \backslash\{0\}$ be arbitrary and let $\tilde{s}=s+R_{x+}$ with $x=\nu(s)$ be its "leading term" in gr $R$. Then we have $\tilde{s} \cdot W \subseteq \tilde{s} \cdot \operatorname{gr} V=\operatorname{gr}(s V)$ and hence, for all positive integers $n$,

$$
(\tilde{s} \cdot W)^{(n)} \subseteq(\operatorname{gr} s V)^{(n)} \subseteq \operatorname{gr}(s V)^{(n)},
$$

where $(s V)^{(n)}$ denotes the $k$-subspace of $R$ generated by the products of length at most $n$ with factors taken from $s V$, and similarly for the other expressions. Therefore,

$$
\operatorname{dim}_{k}(\tilde{s} \cdot W)^{(n)} \leq \operatorname{dim}_{k} \operatorname{gr}(s V)^{(n)}=\operatorname{dim}_{k}(s V)^{(n)} .
$$

To see the latter equality, just note that for any subspace $U \subseteq R$ we have gr $U=$ $\bigoplus_{x \in G} U \cap R_{x} / U \cap R_{x+\cdot}$. Since $s \in R \backslash\{0\}$ was arbitrary, we obtain that

$$
\inf _{s \in R \backslash\{0\}} \varlimsup_{n \rightarrow \infty} \log _{n} \operatorname{dim}_{k}(s V)^{(n)} \geq \inf _{t \in \operatorname{gr}(R) \backslash\{0\}} \varlimsup_{n \rightarrow \infty} \log _{n} \operatorname{dim}_{k}(t W)^{(n)}
$$

and since $W$ was arbitrary, we finally get the desired inequality $\operatorname{Tdeg}_{k}(R) \geq \operatorname{Tdeg}_{k}(\mathrm{gr} R)$.

We remark that in general the above inequality will be strict. For example, let $R=k((X))=k \llbracket X \rrbracket_{X}$ so that $G$ is infinite cyclic and $D=k$. Then $\operatorname{gr} R \cong k\left[X, X_{!}^{-1}\right]$ but $\operatorname{Tdeg}_{k}(R)=\infty$.

Our next goal is to give a (semi-) group theoretical expression for the GKtranscendence degree of $\mathrm{gr} R \cong D * G_{R}$ in the case where $D=k$ and the automorphisms $\alpha(x)\left(x \in G_{R}\right)$ are trivial. Thus we will consider twisted semigroup algebras $k^{\gamma}[H]$ of ordered cancellation semigroups $H$.

Let $H$ be a cancellation semigroup. For any finite subset $E \subseteq H$, we let $E^{(n)}, n \in \mathbb{N}$, denote the set of elements of $H$ that can be written as products of length at most $n$ with factors taken from $E$. Set $d_{E}(n)=$ card $E^{(n)}$ and

$$
t(H)=\sup _{E} \inf _{x \in H} \varlimsup_{n \rightarrow \infty} \log _{n} d_{x E}(n),
$$

where $E$ runs over the finite subsets of $H$ and $x E=\{x e \mid e \in E\}$. Note the formal similarity of this expression with the one defining the GK-transcendence degree of an algebra. Indeed, we have the following result. 
LEMMA 1.3. Let $H$ be an ordered cancellation semigroup and let $k^{\gamma}[H]$ be a twisted semigroup algebra of $H$ over $k$ (with $\gamma: H \times H \rightarrow k^{\bullet}$ a 2-cocycle). Then $\operatorname{Tdeg}_{k}\left(k^{\nu}[H]\right)=$ $t(H)$.

Proof. Fix a finite subset $E \subseteq H$ and let $V \subseteq k^{\gamma}[H]$ be the $k$-subspace generated by $\bar{E}=\{\bar{x} \mid x \in E\} \subseteq k^{\gamma}[H]$. Let $r \in k^{\gamma}[H] \backslash\{0\}$ be arbitrary and let $x=\nu(r)$. For a fixed $n>0$, set $t=d_{x E}(n)$ and let $y_{1}, y_{2}, \ldots, y_{t}$ be the elements of $(x E)^{(n)}$, ordered so that $y_{1}<y_{2}<$ $\ldots<y_{\mathrm{r}}$. Each $y_{i}$ is of the form $y_{i}=x e_{i 1} x e_{i 2} \ldots x e_{i m}$ with $e_{i j} \in E$ and $m \leq n$. We associate with $y_{i}$ the element $\eta_{i}=r \bar{e}_{i 1} r \bar{e}_{i 2} \ldots r \bar{e}_{i m} \in k^{\gamma}[H]$. Note that $\eta_{i} \in(r V)^{(n)}$ and that the lowest terms $\nu\left(\eta_{i}\right)=y_{i}$ are all distinct. Hence the elements $\eta_{1}, \eta_{2}, \ldots, \eta_{t}$ are linearly independent over $k$ and we conclude that $\operatorname{dim}_{k}(r V)^{(n)} \geq t=d_{x E}(n)$. Since $r$ was arbitrary, we further deduce that

$$
\inf _{r \in k^{v}[H \backslash\{0\}} \varlimsup_{n \rightarrow \infty} \log _{n} \operatorname{dim}_{k}(r V)^{(n)} \geq \inf _{x \in H} \varlimsup_{n \rightarrow \infty} \log _{n} d_{x E}(n),
$$

and since $E \subseteq H$ was arbitrary, we obtain the estimate $\operatorname{Tdeg}_{k}\left(k^{\gamma}[H]\right) \geq t(H)$.

To establish the reverse inequality, let $V \subseteq k^{\gamma}[H]$ be a fixed finite-dimensional $k$-subspace and set $E=\bigcup_{r \in V \backslash\{\}}$ Supp $r$, a finite subset of $H$. Now let $x \in H$ be arbitrary and consider $(\bar{x} V)^{(n)} \subseteq k^{\gamma}[H]$. Every nonzero element of $(\bar{x} V)^{(n)}$ has its support in $(x E)^{(n)}$ and we immediately deduce that $\operatorname{dim}_{k}(\bar{x} V)^{(n)} \leq d_{x E}(n)$. As above, this implies that $t(H) \geq$ $\operatorname{Tdeg}_{k}\left(k^{\gamma}[H]\right)$, and the lemma is proved.

Note that the proof of $\operatorname{Tdeg}_{k}\left(k^{\gamma}[H]\right) \leq t(H)$ did not use the ordering of $H$. The following is the main result of this section.

Proposition 1.4. Let $G$ be an ordered group and let $k^{\gamma}[G]$ be a twisted group algebra of $G$ over $k\left(\gamma: G \times G \rightarrow k^{\bullet}\right.$ a 2-cocycle). Assume that $k^{\gamma}[G]$ is Ore and let $k^{\nu}(G)$ denote the classical division ring of fractions of $k^{\gamma}[G]$. Then

$$
\operatorname{Tdeg}_{k}\left(k^{\gamma}(G)\right)=\operatorname{Tdeg}_{k}\left(k^{\gamma}[G]\right)=t(G) \text {. }
$$

Proof. The embedding of $k^{\gamma}[G]$ into the (twisted) Hilbert-Neumann division algebra $k^{\gamma}((G))$ of $G$ over $k$ extends to an embedding of $k^{\gamma}(G)$ into $k^{\gamma}((G)$ ). Hence we may apply Lemmas 1.1 and 1.2 with $R=k^{\gamma}(G)$. Clearly, $G_{R}=G$, as $R$ contains $k^{\gamma}[G]$. Thus we have gr $R \cong k^{\gamma}[G]$, and Lemma 1.2 yields the estimate $\operatorname{Tdeg}_{k}\left(k^{\gamma}(G)\right) \geq \operatorname{Tdeg}_{k}\left(k^{\gamma}[G]\right)$. Since the reverse inequality holds quite generally, we do in fact have equality here. The proposition now follows from Lemma 1.3.

2. Nilpotent groups of class 2 , related results. In this section, we show that, for $G$ a f.g. nilpotent group of class at most two, the invariant $t(G)$ defined in the previous section equals the usual growth degree $d(G)$ of $G$. By $[1], d(G)$ can be computed, for any f.g. nilpotent group $G$, in terms of the descending central series $G=G_{1} \supseteq G_{2} \supseteq \ldots \supseteq G_{c+1}=$ $\langle 1\rangle$, where $G_{i+1}=[G, G$,$] . Namely, d(G)=\sum_{i=1}^{c} i d_{i}$, where $d_{i}=\operatorname{rank}\left(G_{i} / G_{i+1}\right)$.

LEMMA 2.1. Let $G$ be a f.g. nilpotent group of class at most two. Then $t(G)=d(G)$. 
Proof. For any group $G, d(G)$ can be expressed as follows

$$
d(G)=\sup _{E} \varlimsup_{n \rightarrow \infty} \log _{n} d_{E}(n)
$$

where $E$ runs over the finite subsets of $G$ and $d_{E}(n)$ is defined as in the previous section. Thus we always have $t(G) \leq d(G)$. To establish the reverse inequality it suffices to prove the following assertion.

(*) If $E$ is a finite subset of $G$ which generates $G$ as a group then

$$
\varlimsup_{n \rightarrow \infty} \log _{n} d_{E}(n)=d(G) \text {. }
$$

Indeed, suppose $(*)$ is true. Choose a finite subset $E \subseteq G$ which contains 1 and generates $G$ as a group. Then, for any $x \in G$, the set $x E$ also generates $G$ as a group and we conclude from $(*)$ that $\varlimsup_{n \rightarrow \infty} \log _{n} d_{x E}(n)=d(G)$. Therefore, $t(G) \geq d(G)$. Thus it suffices to prove (*) for $G$ f.g. nilpotent of class at most 2 . Set $d_{1}=\operatorname{rank}(G /[G, G]), d_{2}=$ $\operatorname{rank}([G, G])$. If $E$ generates $G$ as a group then there exist elements $e_{1}, e_{2}, \ldots, e_{d_{1}} \in E$ whose images in $G /[G, G]$ are $\mathbb{Z}$-independent. Moreover, $[E, E]$ generates $[G, G]$ as a group and so we can find $\mathbb{Z}$-independent commutators $z_{i}=\left[x_{i}, y_{i}\right]\left(i=1,2, \ldots, d_{2}\right)$ with $x_{i}, y_{i} \in E$. Fix an integer $n \geq 0$. It is easy to check that, for all $k=0,1, \ldots, n^{2}$, the element $\mu_{i, k}=x_{i}^{n} y_{i}^{n} z_{i}^{-k}$ can be written as a product, in some order, with $n$ factors $x_{i}$ and $n$ factors $y_{i}$. For any $d_{2}$-tuple $k=\left(k_{1}, k_{2}, \ldots, k_{d_{2}}\right)$ with $0 \leq k_{i} \leq n^{2}$, set $\mu_{k}=\mu_{1, k_{1}} \mu_{2, k_{2}} \ldots \mu_{d_{2}, k_{k a_{2}}} \in$ $E^{\left(2 d_{2^{n}}\right)}$, and, for any $d_{1}$-tuple $l=\left(l_{1}, l_{2}, \ldots, l_{d_{1}}\right)$ with $0 \leq l_{i} \leq n$, set $\eta_{l}=e_{1}^{l_{1}} e_{2}^{l_{2}} \ldots e_{d_{1}}^{l_{1} \in}$. $E^{\left(d_{1} n\right)}$. Then each $\eta_{1} \mu_{k}$ belongs to $E^{(d n)}$, where $d=d_{1}+2 d_{2}=d(G)$. Moreover, $\eta_{t} \mu_{k}=$ $n_{p} \mu_{q}$ implies $l=p$ and $k=q$ and so we conclude that $d_{E}(d n) \geq(n+1)^{d_{1}}\left(n^{2}+1\right)^{d_{2}}>n^{d}$. Therefore,

$$
\varlimsup_{n \rightarrow \infty} \log _{n} d_{E}(n) \geq \varlimsup_{n \rightarrow \infty} \log _{d n} d_{E}(d n)>d,
$$

which proves our assertion (*), and hence the lemma.

Proposition 1.4 now gives the following corollary.

COROLlaRY 2.2. Let $G$ be a f.g. torsion-free nilpotent group of class at most 2 , let $k^{\nu}[G]$ be a twisted group algebra of $G$ over a field $k$, and let $k^{\nu}(G)$ denote its division ring of fractions. Then $\operatorname{Tdeg}_{k}\left(k^{\gamma}(G)\right)=\operatorname{Tdeg}_{k}\left(k^{\gamma}[G]\right)=d_{k}\left(k^{\gamma}[G]\right)=d(G)$.

For general groups $G$ the invariant $t(G)$ seems to be difficult to handle. In the following lemma we collect a few of its properties. Part (a) is quite easy, and part (b) can be proved by adapting the argument in [1, Lemma 2].

LeMMA 2.3. Let $G$ be any group.

(a) If $H$ is a homomorphic image of $G$ or a subgroup of finite index in $G$ then $t(H) \leq t(G)$.

(b) Let $A$ be a f.g. torsion-free abelian group and let $\langle z\rangle$ be an infinite cyclic group acting rationally irreducibly on $A$. Set $G=A \rtimes\langle z\rangle$, the semidirect product. If $t(G)<\infty$ then some power of $z$ acts trivially on $A$. 
By Proposition 1.4, we have $\operatorname{Tdeg}_{k}(k(G))=\operatorname{Tdeg}_{k}(k[G])$ for any f.g. torsion-free nilpotent group $G$. However, the GK-dimension of $k(G)$ remains mysterious, possibly it is even infinite for $G$ non-abelian. In order to confirm this, it would be enough to prove that $d_{k}(k(G))$ is infinite for the discrete Heisenberg group $G=\langle x, y| z=[x, y]$ is central in $\left.G\right\rangle$, since any non-abelian torsion-free nilpotent group contains a copy of $G$. We close with a result which allows one to control the behavior of GK-dimension under a special, fairly mild sort of localization.

LemMA 2.4. Let $R$ be a $k$-algebra and let $\mathscr{C}$ be a multiplicatively closed central subset of $R$ consisting of regular elements. Let $\alpha$ be a $k$-algebra automorphism of $R$ which maps $\mathscr{C}$ to itself and form the skew Laurent polynomial rings $S=R\left[x^{ \pm 1} ; \alpha\right] \subseteq T=R_{8}\left[x^{ \pm 1} ; \alpha\right]$. Then $d_{k}(T) \leq 2 d_{k}(S)$.

Proof. Let $V \subseteq T$ be a finite-dimensional $k$-subspace. Then $V \subseteq\left\langle W, x, x^{-1}\right\rangle^{(l)}$ for some finite-dimensional $k$-subspace $W \subseteq R$ and some positive integer $l$. Choose $c \in \mathscr{C}$ with $U=c W \subseteq R$ and set $V_{1}=\left\langle W, x, x^{-1}\right\rangle_{k}$ and $U_{1}=\left\langle c, U, x, x^{-1}\right\rangle_{k}$. Then $U_{1}$ is a finitedimensional $k$-subspace of $S$, and $V^{(n)} \subseteq V_{1}^{((n)}$ for all $n$. Now $V_{1}^{(n)}$ is generated by monomials of the form

$$
\mu=x^{i_{0}} c^{-1} u_{1} x^{i_{1}} c^{-1} u_{2} \ldots c^{-1} u_{r} x^{i^{i}}
$$

with $u_{i} \in U, r \geq 0, i_{j} \in \mathbb{Z}$ and $\left(\sum_{j=0}^{r}\left|i_{j}\right|\right)+r \leq n$. By shifting all factors $c^{-1}$ to the right, we can rewrite $\mu$ as follows

$$
\mu=\mu_{1}\left(c^{\alpha^{\alpha_{1}}} c^{\alpha^{t_{2}}} \ldots c^{\alpha^{\prime}}\right)^{-1},
$$

where

$$
\mu_{1}=x^{i_{0}} u_{1} x^{i_{1}} \ldots u_{r} x^{i_{r}} \in U_{1}^{(n)} \quad \text { and } \quad l_{s}=\sum_{i=s}^{r} i_{j} \quad(s=1, \ldots, r)
$$

Now let

$$
\beta_{n}=\prod_{1=-n}^{n} c^{\alpha^{1}}
$$

Then $\mu \beta_{n}^{n}=\mu_{1} \gamma$, where

$$
\gamma=\left(\prod_{s=1}^{r} \beta_{n}\left(c^{\alpha^{\prime}}\right)^{-1}\right) \beta_{n}^{n-r}
$$

and

$$
\begin{aligned}
\beta_{n}\left(c^{\alpha^{\prime}}\right)^{-1} & =\left(x^{n} c x^{-n}\right) \ldots\left(x^{-\left(l_{3}-1\right)} c x^{l_{3}-1}\right)\left(x^{-\left(l_{2}+1\right)} c x^{l_{2}+1}\right) \ldots\left(x^{-n} c x^{n}\right) \\
& =x^{n} c x^{-1} c \ldots x^{-1} c x^{-2} c x^{-1} \ldots c x^{n} \in U_{1}^{(6 n)} .
\end{aligned}
$$

Similarly, $\beta_{n} \in U_{1}^{(6 n+1)}$ and so $\gamma \in U_{1}^{(6 n r+(6 n+1)(n-r))} \subseteq U_{1}^{\left(6 n^{2}+n\right)}$ and $\mu \beta_{n}^{n}=\mu_{1} \gamma \in U_{1}^{\left(6 n^{2}+2 n\right)}$. Since multiplication with $\beta_{n}$ is injective, we conclude that $\operatorname{dim}_{k} V_{1}^{(n)} \leq \operatorname{dim}_{k} U_{1}^{\left(6 n^{2}+2 n\right)}$. The assertion of the lemma now follows easily. 
The following example shows that, in the situation of Lemma $2.4, d_{k}(T)$ will in general be strictly larger than $d_{k}(S)$.

EXAMPLE 2.5. Let $S=k[G]$ be the group algebra of the group $G=\langle x, y| z=[x, y]$ central $\rangle$. Then $S$ can be written as a skew Laurent polynomial ring, $S=R\left[y^{ \pm 1} ; \alpha\right]$ with $R=k\left[x^{ \pm 1}, z^{ \pm 1}\right] \cong k[\mathbb{Z} \oplus \mathbb{Z}]$ and $x^{\alpha}=x z, z^{\alpha}=z$. By Bass' formula, we know that $d_{k}(S)=4$. Let $\mathscr{C}=\left\{1-x z^{m} \mid m \in \mathbb{Z}\right\}$ be the set of all $\alpha$-conjugates of $1-x$ and form $T=R_{\mathscr{C}}[y ; \alpha]$. We claim that $d_{k}(T) \geq 5$. Indeed, consider the subspace $V$ of $T$ generated by $x, y$ and $(1-x)^{-1}$. For a fixed $n$, form products in these elements consisting of $n$ factors $x, n$ factors $y$, and $r$ factors $(1-x)^{-1}(r \leq n)$. One obtains the following elements

$$
y^{n} x^{n} z^{l}\left(1-x z^{m_{1}}\right)^{-1}\left(1-x z^{m_{2}}\right)^{-1} \ldots\left(1-x z^{m_{r}}\right)^{-1},
$$

with $0 \leq l \leq n^{2}, 0 \leq m_{i} \leq n, r \leq n$. In particular, we have the following products

$$
\mu_{1, m, r}=y^{n} x^{n} z^{l}\left(1-x z^{m}\right)^{-1}
$$

with $0 \leq l \leq n^{2}, 0 \leq m \leq n, 1 \leq r \leq n$. It is easily checked that these elements are linearly independent over $k$. Hence the elements $y^{a} \mu_{l, m, r}\left(0 \leq a \leq n, 0 \leq l \leq n^{2}, 0 \leq m \leq n, 1<r<\right.$ $n$ ) are also linearly independent over $k$, and they belong to $V^{(4 n)}$. Therefore, $\operatorname{dim}_{k}$ $V^{(4 n)} \geq n^{5}$ which shows that $d_{k}(T) \geq 5$, as we have claimed.

Addendum (28 March, 1983). I have just received a copy of [6]. Although MakarLimanov only considers the Weyl algebra $A_{1}$ and its field of fractions, his arguments can be conveniently adapted to the group algebra situation to yield the following theorem.

THEOREM. Let $G$ be a torsion-free nilpotent group and assume that $G$ is non-abelian. Then, for any commutative field $k$, the field of fractions $k(G)$ of $k[G]$ contains a free $k$-subalgebra.

In particular, $k(G)$ has infinite GK-dimension over $k$. This answers a question raised in Section 2.

As we have remarked above (in Section 2), it suffices to prove the theorem for the group

$$
G=\langle x, y| z=[x, y] \text { is central in } G\rangle .
$$

We consider a slightly more general situation. Namely, let $K$ be any commutative field containing an element $\lambda \in K^{\circ}$ of infinite order and set $B_{\lambda}=K\left[x^{ \pm 1}, y^{ \pm 1}\right] /(x y-\lambda y x)$. Then $B_{\lambda}$ is a central-simple Noetherian $K$-algebra which is a domain. Clearly, taking $K=k(z)$ and $\lambda=z$, we get $k(G) \cong Q\left(B_{\lambda}\right)$ and so the theorem is a consequence of the following lemma.

Lemma. The $K$-subalgebra of $Q\left(B_{\lambda}\right)$ generated by $a=(1-x)^{-1}$ and $a b=$ $(1-x)^{-1}(1-y)^{-1}$ is free.

This can be proved by embedding $Q\left(B_{\lambda}\right)$ into the skew Laurent power series ring $F_{\lambda}=K(x) *((y))$, where $K(x)$ denotes the field of rational functions over $K$ and with 
multiplication based on the rule $f(x) y=y f(\lambda x)$. Using the identity $b=1+y+y^{2}+\ldots$ in $F_{\lambda}$, one shows that the monomials $a^{i_{\mathrm{o}}} b a^{i_{1}} b \ldots b a^{i_{r+1}}$ with $i_{0}, \ldots, i_{r}>0, i_{r+1} \geq 0$ are linearly independent over $K$. For this, one can closely follow Makar-Limanov, with a few fairly obvious adjustments to the present situation.

Note added in proof (16 March 1984). L. Makar-Limanov has written a note giving a proof of the theorem in the Addendum. For further results on division algebras generated by f.g. nilpotent groups, see the author's article "Group rings and division rings", to appear in the Proceedings of the Antwerp Ring Theory Conference, 1983.

\section{REFERENCES}

1. H. Bass, The degree of polynomial growih of finitely generated nilpotent groups, Proc. London Math. Soc. (3) 25 (1972), 603-614. $1-24$.

2. W. Borho and H. Kraft, Über die Gelfand-Kirillov-Dimension, Math. Ann. 220 (1976),

3. D. R. Farkas, A. H. Schofield, R. L. Snider and J. T. Stafford, The isomorphism question for division rings of group rings, Proc. Amer. Math. Soc. 85 (1982), 327-330.

4. M. Lorenz, Primitive ideals in crossed products and rings with finite group actions, Math. $Z$. 158 (1978) 285-294.

5. M. Lorenz, Division algebras generated by finitely generated nilpotent groups, J. Algebra 85 (1983), 368-381.

6. L. Makar-Limanov, The skew field $D_{1}$ contains free subalgebras, Comm. Algebra 11 (1983), 2003-2006.

7. D. Passman, The algebraic structure of group rings (Wiley-Interscience, 1977).

Max-Planck-Institut für Mathematik

GOTTFRIED-ClAREN-STR. 26

5300 BonN 3, West GERMANY 